\title{
Combined carboplatin and etoposide chemotherapy for patients with recurrent glioma
}

\author{
Jianxin Chen", Yuze Zhao", Xinyu Hou\#, Xiaoyan Gao, Qi Shi, Shan Li, Hongyan Huang^ \\ Department of Oncology, Beijing Shijitan Hospital of Capital Medical University, Beijing, China \\ Contributions: (I) Conception and design: J Chen, H Huang; (II) Administrative support: H Huang; (III) Provision of study materials or patients: \\ J Chen, Y Zhao; (IV) Collection and assembly of data: Y Zhao, X Hou, J Chen; (V) Data analysis and interpretation: X Hou, H Huang; (VI) \\ Manuscript writing: All authors; (VII) Final approval of manuscript: All authors. \\ \#These authors contributed equally to this work. \\ Correspondence to: Hongyan Huang. Department of Oncology, Beijing Shijitan Hospital of Capital Medical University, Beijing, China. \\ Email: huangh1975@mail.ccmu.edu.cn.
}

Background Currently, there is no consensus on the standard of care for patients with recurrent glioma. This study investigated the efficacy of combined carboplatin and etoposide (CE) treatment in recurrent glioma.

Methods: A retrospective analysis was performed on adult patients with recurrent glioma who received combination chemotherapy consisting of CE from September 2017 to November 2020 at the Beijing Shijitan Hospital, Capital Medical University, Beijing. The response rate (RR), defined as the complete response $(\mathrm{CR})+$ partial response (PR), and the disease control rate (DCR), defined as CR + PR + stable disease (SD), were analyzed by Chi-square or Fisher's exact test according to different clinical characteristics. Time to progression (TTP) was estimated using Kaplan-Meier plots and the log-rank test was used to compare differences.

Results: A total of 55 patients were assessed and 47 patients were eligible to be enrolled in this study. There was 1 case of CR (2.1\%), 3 patients with PR (6.4\%), and 18 patients with SD (38.3\%). The RR was $8.5 \%$ and the DCR was $46.8 \%$. When the patients were stratified by World Health Organization (WHO) grade, the DCR was significantly higher in patients with grade 2 and 3 tumors (83.3\% and $50 \%$, respectively) compared to patients with grade 4 tumors $(29.6 \% ; \mathrm{P}=0.039)$. For patients with grade 4 gliomas, the median TTP and median overall survival (OS) were 2 [95\% confidence interval (CI): 0.988 to 3.012] and 7 (95\% CI: 3.626 to 10.374) months, respectively. By comparison, the TTP and OS for patients with grade 2-3 gliomas were 4 (95\% CI: 1.947 to 6.053 ) and 13 (95\% CI: 0.000 to 26.47) months, respectively. The 6-month progression free survival (PFS) and 12-month OS were $11.1 \%$ and $16.3 \%$, respectively, in patients with grade 4 glioma, compared to $37.9 \%$ and $48 \%$, respectively, in patients with grade $2-3$ gliomas.

Conclusions: CE regimen may be effective as a salvage treatment for recurrent glioma. Patients with anaplastic or low-grade glioma may benefit more from such therapy compared to patients with grade 4 tumors.

Keywords! Carboplatin; etoposide; recurrent glioma; survival

Submitted Oct 29, 2021. Accepted for publication Dec 15, 2021.

doi: 10.21037/apm-21-3382

View this article at: https://dx.doi.org/10.21037/apm-21-3382

\footnotetext{
^ ORCID: 0000-0001-8340-6660.
} 


\section{Introduction}

Gliomas are primary malignancies of the central nervous system (CNS), accounting for $80 \%$ of all malignant CNS tumors $(1,2)$. The commonly used World Health Organization (WHO) classification grades tumors from 1-4 according to their presumed cell of origin (astrocytes or oligodendrocytes) (3). Despite much progress in multimodality treatments, relapse or progression is inevitable, especially for high-grade gliomas such as grades 3 and 4 , and partial low grade gliomas with risk factors $(4,5)$. The initial treatment for gliomas includes resection, adjuvant radiotherapy with or without concomitant chemotherapy, and adjuvant chemotherapy of various duration depending on histological types and extent of resection (EOR). The most common chemotherapy for recurrent glioma includes temozolomide (TMZ), nitrosourea, irinotecan and platinum based regimen. However, unlike the putative principles in initial therapy, there is currently no consensus or recommended standard of care for recurrent gliomas because the prognosis of recurrent glioma is poor and the survival benefit conferred by salvage systemic treatment is marginal in most cases (6-8).

While the National Comprehensive Cancer Network (NCCN) supports the use of platin-based therapy for relapsed gliomas, there is no preferred recommendation on the specific combination of drugs (9). Combination treatment consisting of carboplatin and etoposide (CE) is widely used in various malignant tumors including small cell lung cancer (SCLC) (10). However, there is a paucity of consistent data regarding the efficacy of CE therapy in relapsed gliomas (11-13). Therefore, clarification of the efficacy of CE treatment in relapse or progressive glioma patients is urgently warranted.

Although combined carboplatin and etoposide chemotherapy had been used for patients with recurrent glioma in more than one study, the efficacy of the regimen is different in published literatures. In order to clarify the efficacy of this regimen in Chinese patients with recurrent glioma, we performed this retrospective study.

We present the following article in accordance with the STROBE reporting checklist (available at https://dx.doi. org/10.21037/apm-21-3382).

\section{Methods}

\section{Study design and patients}

A retrospective analysis was performed on adult patients with recurrent gliomas who received CE combination chemotherapy (carboplatin plus etoposide) between September 2017 and November 2020 in our clinic in China.

The eligibility criteria for this study included the following: patients aged 18 years and older; histological diagnosis of glioma with WHO grade $2-4$, including glioblastoma multiforme (GBM), diffuse midline glioma, anaplastic astrocytoma (AA), anaplastic oligodendroglioma (AO), anaplastic oligoastrocytoma (AOA), astrocytoma (A), and oligoastrocytoma (OA); and unequivocal recurrent tumor after surgery and radiotherapy or evidence of tumor progression after radiotherapy. Patients with a history of malignancy other than in situ carcinoma of the cervix or non-melanoma skin cancer were ineligible for this study. Patients were followed up routinely by telephone or outpatient consultation.

This study was approved by the Institutional Ethics Committee of Beijing Shijitan Hospital of Capital Medical University (No. 201845). Written informed consent for the research was waived due to the retrospective nature of this study. All procedures performed in this study involving human participants were in accordance with the Declaration of Helsinki (as revised in 2013).

\section{Treatment}

In this study, carboplatin doses were determined to achieve a target area under the curve (AUC) of $5 \mathrm{mg} \cdot \mathrm{mL}^{-1} \cdot \mathrm{min}$ and glomerular filtration rate (GFR), according to the Calvert formula: Dose $=$ AUC(GFR+25), and etoposide $\left(60 \mathrm{mg} / \mathrm{m}^{2}\right.$ per day for 3 to 5 days) were delivered intravenously each cycle. Generally, the $\mathrm{CE}$ regimen was administered as follows: carboplatin 100-200 $\mathrm{mg}$ on days 1-4 and etoposide $60 \mathrm{mg} / \mathrm{m}^{2}$ on days $1-5$, every 4 weeks. Carboplatin was administered by a 30-minute infusion followed by a 60-minute infusion of etoposide. Doses in subsequent cycles were reduced or the interval between cycles were lengthened due to hematological toxicities.

\section{Statistical analyses}

Overall survival (OS) was measured from the first day of application of chemotherapy to the date of death or last day of follow-up for patients who had not progressed at the date of analysis. Time to progression (TTP) was defined as the interval from chemotherapy commencement to date of progression.

Response was assessed using the Response Assessment 
Table 1 Basic patient characteristics

\begin{tabular}{|c|c|}
\hline Characteristics & $\mathrm{n}(\%)$ \\
\hline \multicolumn{2}{|l|}{ Age (years) } \\
\hline$<60$ & $43(91.5)$ \\
\hline$\geq 60$ & $4(8.5)$ \\
\hline \multicolumn{2}{|l|}{ Gender } \\
\hline Male & $26(55.3)$ \\
\hline Female & $21(44.6)$ \\
\hline \multicolumn{2}{|c|}{ World Health Organization (WHO) grade } \\
\hline 2 & $6(12.8)$ \\
\hline 3 & $14(29.8)$ \\
\hline 4 & $27(57.4)$ \\
\hline \multicolumn{2}{|c|}{ Temozolomide (TMZ) adjuvant therapy } \\
\hline Yes & $40(85.1)$ \\
\hline No & $7(14.9)$ \\
\hline \multicolumn{2}{|c|}{ Surgery after recurrence } \\
\hline Yes & $19(40.4)$ \\
\hline No & $28(59.6)$ \\
\hline \multicolumn{2}{|c|}{ Salvage TMZ treatment } \\
\hline Yes & $32(68.1)$ \\
\hline No & $15(31.9)$ \\
\hline \multicolumn{2}{|l|}{ Bevacizumab } \\
\hline Yes & $24(51.1)$ \\
\hline No & $23(48.9)$ \\
\hline \multicolumn{2}{|c|}{ Salvage nitrosourea } \\
\hline Yes & $1(2.1)$ \\
\hline No & $46(97.9)$ \\
\hline \multicolumn{2}{|c|}{ Salvage chemotherapy lines } \\
\hline 1 & $14(29.8)$ \\
\hline 2 & $32(68.1)$ \\
\hline 3 & $1(2.1)$ \\
\hline
\end{tabular}

in Neuro-Oncology (RANO) criteria. The response rate (RR) was defined as the complete response (CR) + partial response (PR), and the disease control rate (DCR) was defined as $\mathrm{CR}+\mathrm{PR}+$ stable disease $(\mathrm{SD})$. The RR and DCR were analyzed by Chi-square or Fisher's exact test according to different clinical characteristics. The KaplanMeier method was used in the analysis of TTP and OS.
The log-rank test was performed to compare the difference in OS or TTP.

\section{Results}

\section{Patient characteristics}

A total of 55 patients were considered for this study. Treatment was terminated for 8 patients after 1 chemotherapy course for various personal reasons, and a total of 47 patients were finally enrolled in this study. The characteristics of the patients are summarized in Table 1. Among the 47 patients, $55.3 \%$ were male and the median age was 45 years (range, 12-89 years). Histologically, there were 25 cases of glioblastoma (GBM), 2 patients had diffuse midline glioma, 14 patients presented with $\mathrm{AA}, 10$ patients had anaplastic oligo-astrocytoma (AOA), 5 had diffuse astrocytoma (DA) at the time of surgery, and 9 presented with GBM from the initial grade 2 or 3 tumor. All patients received surgery followed by radiotherapy. Temozolomide (TMZ) was administered to $87 \%$ of patients due to relapse or progression of disease.

\section{Chemotherapy}

After surgery and radiotherapy, all patients with a recurrence or residual disease in progression were treated with CE regimen for 3-4 days, every 4 weeks. A total of 174 chemotherapy courses were administered with a median number of 3 cycles per patient (range, 1-22 cycles). There was 1 case of CR (2.1\%), 3 cases of PR (6.4\%), and 18 patients with SD (38.3\%). The RR (CR + PR) was $8.5 \%$ and the DCR $(\mathrm{CR}+\mathrm{PR}+\mathrm{SD})$ was $46.8 \%$. A total of 25 patients (53.2\%) experienced progressive disease (PD).

In patients who had grade 4 gliomas, no CR was observed, 3 patients had PR (11.1\%), and 6 had SD (22.2\%). The RR was $11.1 \%$ and the DCR was $33.3 \%$, and more than a half of the patients had PD (66.7\%).

In patients who had grade 3 gliomas, the best response was SD, which was observed in 6 patients. In grade 2 glioma patients, 1 CR was observed, 4 patients had SD, and 1 patient had PD.

The above results demonstrated that the DCR was higher in patients with grade 2-3 tumors compared to patients with grade 4 tumors (Table 2). There was no correlation between DCR and age, surgery, salvage chemotherapy line, nor adjuvant chemotherapy. There was no association between $\mathrm{RR}$ and any of the clinical parameters examined (data not 
Table 2 The RR and DCR in patients stratified by WHO grade

\begin{tabular}{lccccccc}
\hline WHO grade & $\mathrm{n}$ & $\mathrm{RR}(\%)$ & Chi-square tests value & $\mathrm{P}$ & $\mathrm{DCR}(\%)$ & Chi-square tests value & $\mathrm{P}$ \\
\hline 2 & 6 & 16.7 & 3.117 & 0.21 & 83.3 & 6.479 \\
3 & 14 & 0 & - & - & 50 & - & - \\
4 & 27 & 11.1 & - & - & 29.6 & - & - \\
\hline
\end{tabular}

WHO, World Health Organization; RR, response rate; DCR, disease control rate.

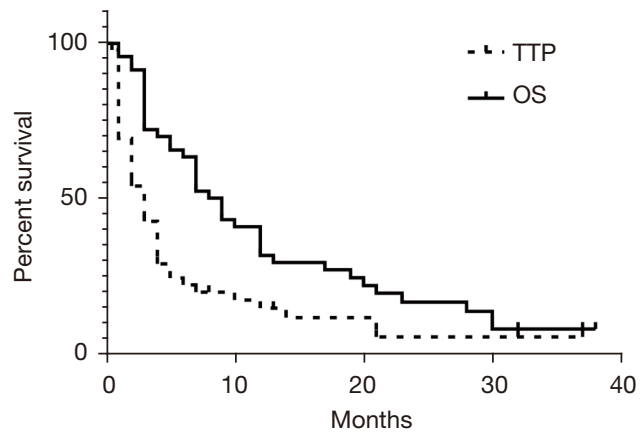

Figure 1 Kaplan-Meier estimation of the time to progression (TTP) and the overall survival (OS) in patients with recurrent glioma.

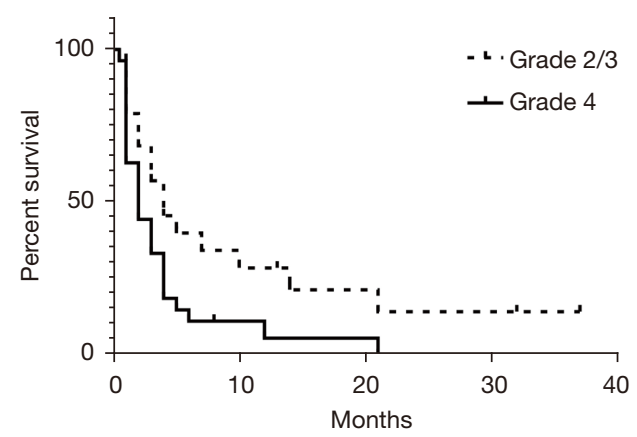

Figure 2 Kaplan-Meier estimation of the time to progression (TTP) in patients with recurrent glioma stratified by World Health Organization (WHO) grade.

shown).

\section{Progression and survival}

At the time of this report, a total of 40 deaths (85.1\%) were recorded. The median TTP for all 47 patients was 3 months (95\% CI: 1.905 to 4.095 months) and the median OS was 9 months (95\% CI: 6.844 to 11.156 months; Figure 1). The PFS at 6 months and the OS at 12 months were $22.2 \%$ and

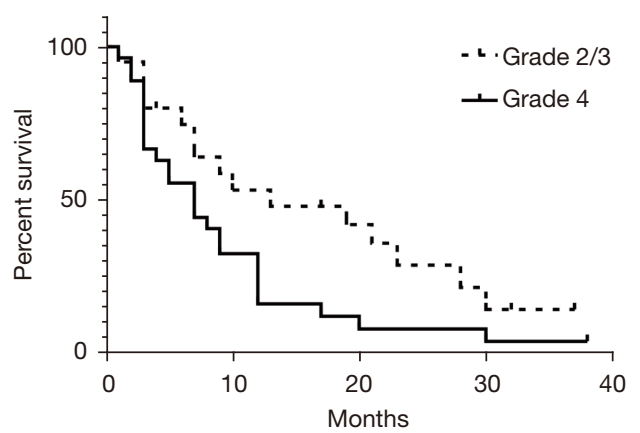

Figure 3 Kaplan-Meier estimation of the overall survival (OS) in patients with recurrent glioma stratified by World Health Organization (WHO) grade.

\section{$32.1 \%$, respectively.}

When the patients were stratified by WHO grade of tumor, both TTP and OS were significantly shorter in patients with grade 4 tumors compared to patients with grade $2-3$ tumors $(\mathrm{P}<0.05$; Figures 2,3$)$.

The PFS at 6 months (PFS 6) was estimated using the Kaplan-Meier method and the results indicated that PFS6 in grade 4 glioma patients $(11.1 \%)$ was significantly lower than that in grade $2-3$ patients $(37.9 \%)$. The OS at 12 months was calculated as an endpoint. The 1 -year survival in patients with grade 4 tumors was $16.3 \%$ compared to $48 \%$ in patients with grade $2-3$ tumors (Table 3 ).

\section{Discussion}

Currently, salvage treatment options for patients with recurrent glioma are limited (14). Bevacizumab was originally approved for the treatment of recurrent GBM and then subsequently, for the treatment of recurrent high-grade gliomas $(15,16)$. However, in China, the costs of bevacizumab are not covered by the public insurance system and therefore, it represents a significant financial burden for some patients $(17,18)$. TMZ and nitrosourea are commonly recommended after disease progression or 
Table 3 Kaplan-Meier estimation of the median time to progression and median overall survival in patients stratified by WHO grade

\begin{tabular}{|c|c|c|c|c|c|c|c|}
\hline WHO grade & $\mathrm{n}$ & \multicolumn{3}{|c|}{ OS } & \multicolumn{3}{|c|}{ TTP } \\
\hline $2-3$ & 20 & 13 & $0.000-26.470$ & 0.036 & 4 & $1.947-6.053$ & 0.048 \\
\hline 4 & 27 & 7 & $3.626-10.374$ & _- & 2 & $0.988-3.012$ & - \\
\hline
\end{tabular}

WHO, World Health Organization; mTTP, median time to progression; mOS, median overall survival; $\mathrm{Cl}$, confidence interval.

relapse as salvage chemotherapy due to its definitive efficacy (4,19-21). However, nitrosourea is unavailable in China and TMZ resistance is inevitable due to its wide application in the adjuvant setting $(22,23)$. Although other cytotoxic agents such as irinotecan have been used in recurrent GBM with moderate efficacy, alterative chemotherapy regimen for relapsed gliomas is still limited (24). Therefore, exploring novel regimens or examining existed therapies that are used in other malignant tumors, is crucial for the salvage treatment of recurrent glioma.

Carboplatin is a 'second-generation' cytotoxic platinum complex structurally related to cisplatin, and is widely used in ovarian cancer and SCLC. Etoposide is a podophyllotoxin derivative which delays progression of the cell cycle through the late S or early G2 phase, it is a potent anti-tumor drug that belongs to the class of topoisomerase poisons. In this current study, the response rate of CE regimen in recurrent gliomas was rather low, with SD presenting as the best response observed in the entire cohort. Interestingly, the efficacy of CE therapy differed depending on the grade of the tumor. For patients with grade 4 gliomas, CE regimen resulted in lower disease control rate and shorter TTP or OS compared to patients with grade $2-3$ tumors (4 vs. 2 months, $\mathrm{P}=0.028 ; 13$ vs. 7 months, $\mathrm{P}=0.036$ ). In patients with recurrent grade 4 gliomas who received CE salvage chemotherapy, the estimated PFS6 and 12-month OS was $11.1 \%$ and $16.3 \%$, which failed to show any obvious advantage compared to dose intensive TMZ treatment in recurrent GBM patients as demonstrated in the RESCUE study (21). Furthermore, in patients with anaplastic glioma, the estimated PFS6 (40.8\%) and OS (55\%) in this study was similar to that reported in the RESCUE study $(35.7 \%$ and $60.7 \%$, respectively).

In this study, the efficacy of CE regimen in recurrent GBM was poor which was consistent with previous report (11). However, the efficacy of CE regime was better in patients with recurrent glioma of grade 2 or 3 compared with recurrent GBM. In this study cohort, there were only 6 patients with grade 2 tumors, and the estimated PFS6
(16.7\%) and 12-month OS (50\%) data should be interpreted with caution due to the limited sample size. Notably, CR was observed in 1 patient with grade 2 tumor after 2 cycles of $\mathrm{CE}$ chemotherapy, suggesting that salvage $\mathrm{CE}$ regimen may be effective for patients with grade 2 recurrent gliomas. Nonetheless, the preliminary results in this study suggested that $\mathrm{CE}$ regimen may confer a superior clinical benefit in patients with grade 2-3 tumors compared to patients with grade 4 tumors following disease progress or recurrence after initial surgery and adjuvant therapy

Since CE regimen has been widely used in different malignant tumors for decades and the reported related adverse events is similar in different diseases $(11,13,25)$, toxicity was not a primary endpoint in this study. However, hematological toxicity was the most common adverse event observed in this study cohort and most patients received granulocyte-colony stimulating factor (GSF) treatment during the course of chemotherapy. It should be noted that grade 5 toxicity was observed in 1 patient who died from serious neutropenia and pneumonia. Therefore, the Eastern Cooperative Oncology Group (ECOG) score and bone marrow function should be monitored prior to administration of CE therapy. Treatmentrelated hepatotoxicity was generally less common than myelosuppression and there was no serious hepatotoxicity observed in this study.

There were some limitations in this study. First, the retrospective nature of this study may be a source of potential bias and may exclude meaningful comparisons and conclusions to some extent. Second, the limited sample size may contribute to bias. Moreover, the molecular signature of the tumor was not provided in the analysis due to the limited sample size. Future well-designed prospective studies with larger sample sizes should be performed to further validate the preliminary results of this investigation.

\section{Conclusions}

CE regimen may be an effective salvage treatment for 
recurrent gliomas. Patients with anaplastic or lowgrade gliomas may derive more benefit from CE therapy compared to patients with grade 4 tumors.

\section{Acknowledgments}

Funding: This work was funded by the National Natural Science Foundation of China (81572799).

\section{Footnote}

Reporting Checklist: The authors have completed the STROBE reporting checklist. Available at https://dx.doi. org/10.21037/apm-21-3382

Data Sharing Statement: Available at https://dx.doi. org/10.21037/apm-21-3382

Conflicts of Interest: All authors have completed the ICMJE uniform disclosure form (available at https://dx.doi. org/10.21037/apm-21-3382). The authors have no conflicts of interest to declare.

Ethical Statement: The authors are accountable for all aspects of the work in ensuring that questions related to the accuracy or integrity of any part of the work are appropriately investigated and resolved. This study was approved by the Institutional Ethics Committee of Beijing Shijitan Hospital of Capital Medical University (No. 201845). Written informed consent for the research was waived due to the retrospective nature of this study. All procedures performed in this study involving human participants were in accordance with the Declaration of Helsinki (as revised in 2013).

Open Access Statement: This is an Open Access article distributed in accordance with the Creative Commons Attribution-NonCommercial-NoDerivs 4.0 International License (CC BY-NC-ND 4.0), which permits the noncommercial replication and distribution of the article with the strict proviso that no changes or edits are made and the original work is properly cited (including links to both the formal publication through the relevant DOI and the license). See: https://creativecommons.org/licenses/by-nc-nd/4.0/.

\section{References}

1. Kohler BA, Ward E, McCarthy BJ, et al. Annual report to the nation on the status of cancer, 1975-2007, featuring tumors of the brain and other nervous system. J Natl Cancer Inst 2011;103:714-36.

2. Yin CL, Lv SQ, Chen XY, et al. The role of glioma stem cells in glioma tumorigenesis. Front Biosci (Landmark Ed) 2014;19:818-24.

3. Louis DN, Ohgaki H, Wiestler OD, et al. The 2007 WHO classification of tumours of the central nervous system. Acta Neuropathol 2007;114:97-109.

4. Chelliah SS, Paul EAL, Kamarudin MNA, et al. Challenges and Perspectives of Standard Therapy and Drug Development in High-Grade Gliomas. Molecules 2021;26:1169.

5. $\mathrm{Xu} \mathrm{F}, \mathrm{Gao} \mathrm{Y}, \mathrm{Ni}$ W, et al. Patterns of local failure in patients with high-grade glioma after postoperative radiotherapy with or without chemotherapy. Transl Cancer Res 2019;8:985-91.

6. Chen-Zhao X, Aznar-García L. Diagnosis and management of spinal metastasis of primary brain tumours. AME Case Rep 2018;2:26.

7. Bush NA, Chang SM, Berger MS. Current and future strategies for treatment of glioma. Neurosurg Rev 2017;40:1-14.

8. Stupp R, Brada M, van den Bent MJ, et al. High-grade glioma: ESMO Clinical Practice Guidelines for diagnosis, treatment and follow-up. Ann Oncol 2014;25 Suppl 3:iii93-101.

9. Nabors LB, Portnow J, Ammirati M, et al. NCCN Guidelines Insights: Central Nervous System Cancers, Version 1.2017. J Natl Compr Canc Netw 2017;15:1331-45.

10. Wang S, Zimmermann S, Parikh K, et al. Current Diagnosis and Management of Small-Cell Lung Cancer. Mayo Clin Proc 2019;94:1599-622.

11. Tonder M, Weller M, Eisele G, et al. Carboplatin and Etoposide in Heavily Pretreated Patients with Progressive High-Grade Glioma. Chemotherapy 2014;60:375-8.

12. Franceschi E, Lamberti G, Paccapelo A, et al. Third-line therapy in recurrent glioblastoma: is it another chance for bevacizumab? J Neurooncol 2018;139:383-8.

13. Franceschi E, Cavallo G, Scopece L, et al. Phase II trial of carboplatin and etoposide for patients with recurrent highgrade glioma. Br J Cancer 2004;91:1038-44.

14. Ghiaseddin AP, Shin D, Melnick K, et al. Tumor Treating Fields in the Management of Patients with Malignant Gliomas. Curr Treat Options Oncol 2020;21:76.

15. Nguyen HT, Nguyen N, Liu LY, et al. Bevacizumab at first recurrence after standard radio-chemotherapy is associated with improved overall survival in glioblastoma 
patients with large tumor burden. Neurooncol Pract 2019;6:103-11.

16. Lim Fat MJ, Maurice C, Maganti M, et al. Bevacizumab in Recurrent High-Grade Gliomas: A Canadian Retrospective Study. Can J Neurol Sci 2018;45:56-61.

17. Diao Y, Qian J, Liu Y, et al. How government insurance coverage changed the utilization and affordability of expensive targeted anti-cancer medicines in China: an interrupted time-series study. J Glob Health 2019;9:020702.

18. Zhang Y, Wushouer H, Han S, et al. The impacts of government reimbursement negotiation on targeted anticancer medication price, volume and spending in China. BMJ Glob Health 2021;6:e006196.

19. Chang S, Zhang P, Cairncross JG, et al. Phase III randomized study of radiation and temozolomide versus radiation and nitrosourea therapy for anaplastic astrocytoma: results of NRG Oncology RTOG 9813. Neuro Oncol 2017;19:252-8.

20. Paccapelo A, Lolli I, Fabrini MG, et al. A retrospective pooled analysis of response patterns and risk factors in recurrent malignant glioma patients receiving

Cite this article as: Chen J, Zhao Y, Hou X, Gao X, Shi Q, Li S, Huang H. Combined carboplatin and etoposide chemotherapy for patients with recurrent glioma. Ann Palliat Med 2021;10(12):12650-12656. doi: 10.21037/apm-21-3382 a nitrosourea-based chemotherapy. J Transl Med 2012;10:90.

21. Perry JR, Bélanger K, Mason WP, et al. Phase II trial of continuous dose-intense temozolomide in recurrent malignant glioma: RESCUE study. J Clin Oncol 2010;28:2051-7.

22. Lee SY. Temozolomide resistance in glioblastoma multiforme. Genes Dis 2016;3:198-210.

23. Johannessen TC, Bjerkvig R. Molecular mechanisms of temozolomide resistance in glioblastoma multiforme. Expert Rev Anticancer Ther 2012;12:635-42.

24. Lieberman FS, Wang M, Robins HI, et al. Phase 2 Study of Radiation Therapy Plus Low-Dose Temozolomide Followed by Temozolomide and Irinotecan for Glioblastoma: NRG Oncology RTOG Trial 0420. Int J Radiat Oncol Biol Phys 2019;103:878-86.

25. Lieu C, Chow L, Pierson AS, et al. A phase I study of bortezomib, etoposide and carboplatin in patients with advanced solid tumors refractory to standard therapy. Invest New Drugs 2009;27:53-62.

(English Language Editor: J. Teoh) 